\title{
Session 11: Biorefining Technology Deployment and Demonstration
}

\author{
Jeff Tolan
}

Published online: 28 March 2009

(C) Humana Press 2009

This session featured a wide range of presentations and posters relating to commercially targeted areas of biomass conversion. The scale of the efforts includes laboratory, pilot, and demonstration, as well as process designs. The feedstocks range from agricultural sources such as wheat straw and rice straw to wood chips and sugar cane bagasse. The products made include ethanol, other alcohols and chemicals, and solid products such as paper pulp. The high quality and broad scope of the presentations is an indication of the progress and interest in this field.

Mark Holtzapple, Texas A and M University, presented "MixAlco Process: Fuels and Chemicals from Biomass". The MixAlco process can use a wide range of feedstocks; the presentation focused on sugar cane bagasse. The feedstock is contacted with lime and air to enhance the digestibility. The lime-treated feedstock is then subjected to a mixed culture of aid-forming marine microorganisms. The acids are neutralized with calcium carbonate or ammonium carbonate to form the carboxylic acids. The carboxylate salts are concentrated by vapor compression evaporation and can then be chemically converted to a variety of products, including carboxylic acids, primary alcohols including ethanol, ketones from thermal conversion, secondary alcohols, or hydrocarbons. A demonstration plant is under construction for this process.

Mark Yancey, BBI International, presented "BBI International Cellulosic Ethanol Project Update". BBI International is engaged in activities relating to the engineering design and development of commercial cellulosic ethanol plants. BBI is currently designing plants to produce 4-12 million gallons per year of ethanol. The feedstocks are agricultural products and wastes. The designs address the challenges of biomass pretreatment, fermentation, and debt financing.

P. Kaparaju, University of Copenhagen, Denmark, presented "Bioethanol, Biohydrogen, and Biogas Production from Wheat straw and its Byproducts in a Biorefinery Concept". This process uses wheat straw as the feedstock, which was subjected to pretreatment at $195^{\circ} \mathrm{C}$ for $10-15 \mathrm{~min}$. This resulted in a cellulose-rich fraction, which was subjected to

J. Tolan $(\bowtie)$

Iogen Corporation, Ottawa, ON, Canada

e-mail: jefft@iogen.ca 
enzymatic hydrolysis and fermentation to ethanol, and a hemicellulose-rich fraction, which was fermented anaerobically to hydrogen. The stillage was subsequently fermented to methane.

Wim Soetaert, University of Ghent, Belgium, and Jonathan Mielenz, Oak Ridge National Laboratory, presented "Integrated Biorefining at Ghent Bio-Energy Valley". Ghent Bio-Energy Valley is a public-private partnership aiming to support the development of sustainable bio-based activities in the region of Ghent, Belgium. Currently, several biofuel projects are already operational or under construction in the port of Ghent. These initiatives are turning Ghent into the prime bioport of Europe.

Brian Foody, Iogen Corporation, presented "Status of Iogen's Process for Production of Ethanol from Cellulose". Iogen Corporation has been operating a demonstration plant for the conversion o wheat straw to ethanol since 2004. The process includes a pretreatment with steam and dilute sulfuric acid, followed by enzymatic hydrolysis of the cellulose, and then fermentation of the glucose and xylose to ethanol by using recombinant Sacchaomyces. The ethanol is finished as E85 fuel for commercial and fleet use. The demonstration plant is the final step in scale-up of the process prior to commercial operation. This presentation described the operation of the demonstration plant to date and Iogen's plans for deploying commercial plants that produce ethanol from cellulose.

J. Destain, University of Gembloux, Belgium, presented a poster titled "Bioethanol Subproducts as a Basis of a Plant Biorefinery". This research focuses on the behavior of nonsugar compounds during the conversion of biomass to ethanol. With sugar beets as the feedstock, the nonsugar compounds include vinasse, betaine, saponins, and raffinose. These compounds offer opportunities for recovery as useful products and for chemical conversion to other compounds.

J. Helmeris, Lulea University of Technology, Sweden, presented a poster titled "Production of Value-Added Chemicals from Xylan Extraction in a Kraft Pulp Mill and the Effect on Pulp Quality". In the Kraft process, hemicelluloses are lost in the cooking to the black liquor stream, which is subsequently burned in the recovery boiler. The hemicellulose is of low fuel value, so recovery of the chemical prior to burning represents an opportunity to increase revenues. Extraction of the wood chips with hot alkali was shown to remove the xylan from the wood chips. The xylan can then be hydrolyzed to xylose and fermented to succinic acid. The pulp made from the wood chips after extraction showed no compromises in quality.

M. Petersen, DONG Energy, presented a poster titled "The IBUS Process-Large Scale SSF at High Dry Matter Content $(>25 \%)$ ". This is a process for ethanol production that uses many of the conventional steps, but carried out at high solids-low water conditions. This minimizes the consumption of water and energy. The use of high dry matter concentrations provides challenges in viscosity, agitation, and mass transfer. The data presented in this poster indicate that similar results can be obtained in low and high dry matter conditions. 